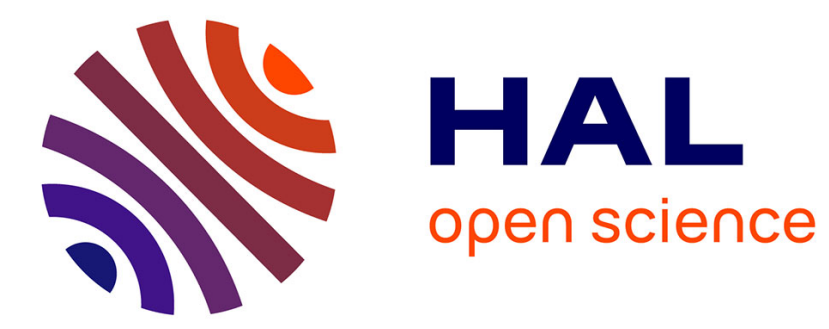

\title{
Valoriser le quartier par la diversité religieuse
}

Victor Albert Blanco

\section{To cite this version:}

Victor Albert Blanco. Valoriser le quartier par la diversité religieuse: Regards croisés entre la Goutte d'Or (Paris) et El Raval (Barcelone). CIST2018 - Représenter les territoires / Representing territories, Collège international des sciences territoriales (CIST), Mar 2018, Rouen, France. pp.478-483. hal01854388

\section{HAL Id: hal-01854388 \\ https://hal.science/hal-01854388}

Submitted on 6 Aug 2018

HAL is a multi-disciplinary open access archive for the deposit and dissemination of scientific research documents, whether they are published or not. The documents may come from teaching and research institutions in France or abroad, or from public or private research centers.
L'archive ouverte pluridisciplinaire HAL, est destinée au dépôt et à la diffusion de documents scientifiques de niveau recherche, publiés ou non, émanant des établissements d'enseignement et de recherche français ou étrangers, des laboratoires publics ou privés. 


\title{
Valoriser le quartier par la diversité religieuse Regards croisés entre la Goutte d'Or (Paris) et El Raval (Barcelone)
}

\author{
AUTEUR \\ Víctor ALBERT BLANCO
}

\section{RÉSUMÉ}

Accueillant historiquement des populations fragiles socialement, les quartiers de la Goutte d'Or et d'El Raval ont été longtemps stigmatisés. Dans le but affiché de renverser cette situation, les administrations ont programmé des transformations importantes. Plus visibles dans l'urbanisme et le logement, ces actions ont atteint aussi d'autres domaines. Elles ont facilité un processus de gentrification et ont été la base d'une revalorisation de l'image de ces quartiers. La diversité culturelle et religieuse a été insérée dans ce processus, convertie en objet de promotion institutionnelle. Bien que les manifestations de cette diversité ont été, et sont toujours, à l'origine de conflits inscrits dans les rapports sociaux d'altérité et de subordination, la mémoire collective est réinterprétée pour satisfaire le processus de revalorisation. Cette communication interroge ces discours et ces politiques à partir de données issues d'une enquête menée dans les deux quartiers.

\section{MOTS CLÉS}

Quartiers, diversité, religions, minorités, espace public, mémoire

\begin{abstract}
Historically welcoming socially fragile populations, the neighbourhoods of Goutte d'Or and EI Raval have long been stigmatised. With the stated aim of reversing this situation, administrations have planned important transformations. More visible in the urban and housing field, government actions have also reached other areas, facilitated a process of gentrification and been the basis for a revaluation of the image of these neighbourhoods. Cultural and religious diversity has been inserted in this process and converted into an object of institutional promotion. Although the manifestations of this diversity have been, and still are, causes for conflicts inscribed within social relations of otherness and subordination, the collective memory is reinterpreted to satisfy the process of revalorisation. This paper examines these discourses and policies on the basis of data from a survey conducted in both neighbourhoods.
\end{abstract}

\section{KEYWORDS}

Neighbourhood, Diversity, Religions, Minorities, Public space, Memory

\section{INTRODUCTION}

Quartier populaire de Paris, avec plus de $40 \%$ d'habitants se déclarant employés ou ouvriers en 2016, la Goutte d'Or est aussi une « centralité immigrante " ${ }^{1}$ depuis la deuxième moitié du $X X^{\mathrm{e}}$ siècle (Toubon \& Messamah, 1990). Les administrations publiques ont entamé, à partir des années 80 , des fortes interventions dans le but affiché de lutter contre la dégradation urbaine. À Barcelone, El Raval est aussi souvent défini comme un quartier populaire. La structure de son habitat et sa situation géographique ont permis qu'il devienne le point de destin de plusieurs vagues d'immigration. La population du quartier a été historiquement très précarisée. Encore aujourd'hui, pour un taux du revenu moyen de la ville de Barcelone de 100,

1 L'APUR montre aussi que $36 \%$ des habitants du quartier sont nés à l'étranger, contre $20 \%$ dans Paris. 
il est de $65,9^{2}$ dans le quartier. De même, El Raval compte la plus forte présence de population étrangère de la ville : $48,5 \%$ des habitants de ce territoire ont une nationalité étrangère ${ }^{3}$ (16,6\% pour l'ensemble de Barcelone). Les pouvoirs locaux lancent la rénovation urbaine du quartier au même moment qu'à Paris.

À El Raval comme à la Goutte d'Or, les discours véhiculant une image stigmatisée ont légitimé les interventions urbanistiques programmées. Dans les deux quartiers, la mixité sociale devient un objectif politique, ce qui se traduit par l'arrivée de nouveaux habitants plus aisés. Un processus de gentrification commence alors sur ces deux territoires, bien que ses effets restent encore mitigés quant à leur configuration sociodémographique (Bacqué, 2006 ; Fernandez, 2014 ; Horta, 2010 ; Milliot, 2015).

L'étude de la gentrification se focalise souvent sur l'évolution des données sociodémographiques, du prix immobilier ou de la progressive transformation commerciale. Certains travaux portent aussi un regard sur les discours médiatiques et politiques qui prétendent revaloriser les quartiers concernés, car ils jouent un rôle déterminant dans ces processus (Bacqué, 2006). Ces discours portent souvent sur les résultats, attendus ou réels, de ces transformations. Les administrations insistent sur les changements que leurs actions amèneront dans le quartier et notamment sur la disparition de phénomènes perçus comme problématiques. Les médias participent aussi de la reproduction de ces discours et mettent en avant les changements urbains et les nouveaux établissements commerciaux ou culturels qui y fleurissent.

Dans ce processus, la mémoire du quartier est souvent réinterprétée et mise au service du projet de revalorisation. Néanmoins, comme le note Barbara Morovich, " une contradiction apparaît, car on souhaite opérer un travail sur la mémoire de lieux qu'on efface » (2014). Dans cette communication, la mémoire revalorisée porte moins sur les "lieux » que sur les manifestations religieuses et culturelles de groupes minorisés et stigmatisés qui habitent ces quartiers.

\section{LA DIVERSITÉ RELIGIEUSE POSTMIGRATOIRE}

La diversité, culturelle et religieuse d'El Raval et de la Goutte d'Or a été souvent posée comme problématique par les discours dominants, insistant sur les difficultés à la gérer et sur les éléments négatifs qui y seraient associés. Néanmoins, d'autres discours émergent au moment où la transformation urbaine commence. La diversité devient alors un élément positif.

L'émergence de cette diversité est corrélée à l'immigration débutée dans ces quartiers au cours de la deuxième moitié du $\mathrm{XX} \mathrm{X}^{\mathrm{e}}$ siècle. Les cultes minoritaires n'existent que depuis quelques décennies, mais certaines de leurs expressions sont perçues de manière disruptive et provoquent de fortes polémiques, même à l'échelle nationale. La plupart de ces controverses portent sur l'islam et les musulmans et, comme le note Nilüfer Göle, « il ne s'agit pas alors de simples phénomènes médiatiques, car ces controverses sont liées à des événements précis, se déroulent dans des lieux spécifiques et impliquent des citoyens musulmans et non musulmans. Chaque événement controverse crée un champ public, fait apparaître différents acteurs, mobilise une certaine rhétorique et déploie un répertoire d'action particulier » (2015:20).

2 Source : « El Raval. Ciutat Vella » juillet 2016, publié par la mairie de Barcelone [en ligne : www.bcn. cat/estadistica/catala/documents/barris/01_CV_Raval_2016.pdf].

3 Source : " La població estrangera a Barcelona », janvier 2016, publié par la mairie de Barcelone [en ligne : www.bcn.cat/estadistica/catala/dades/inf/pobest/pobest16/pobest16.pdf]. 
Plusieurs exemples illustrent cette situation dans les quartiers concernés par cette communication. À la Goutte d'Or, en raison du manque d'espace dans les deux salles de prière du quartier, les fidèles avaient pris, depuis la fin des années 90, l'habitude de prier dans la rue. Cette situation a provoqué l'intervention de plusieurs acteurs et le ministère de l'Intérieur a interdit en 2010 de prier dans la rue. Bien que la controverse acquière une dimension nationale, le débat reste ancré dans le local et suscite des réactions contradictoires. Certains habitants non musulmans considèrent ce phénomène comme une occupation illégitime de l'espace public, et l'interprètent par le biais d'une frontière symbolique (Lamont, 2000) qui l'oppose aux valeurs républicaines et à la laïcité. Pour une partie des musulmans, prier dans la rue est en revanche corrélé à leur condition minoritaire et infériorisée au sein de la société française, conséquence de la précarité de leurs lieux de culte. Pour certains, il s'agit aussi de l'occasion d'être visibles et de réclamer leur droit à la cité en ayant des espaces de culte dignes.

À son tour, El Raval abrite une bonne partie des salles de prière musulmanes de Barcelone. Au moins 6 des 29 mosquées de la ville y sont localisées. La presse montre que certaines ont dû faire face, au moment de leur ouverture, à l'opposition de voisins. Des tensions continuent aujourd'hui : le propriétaire d'un bar se plaint que les fidèles musulmans occupent le trottoir en face de son établissement, un autre, qui détient un appartement touristique, a empêché l'ouverture d'une salle de prière dans les locaux en rez-de-chaussée du bâtiment. Néanmoins, les polémiques les plus médiatisées sont survenu après l'intervention d'acteurs extérieurs au quartier. Par exemple, lors de la campagne des élections municipales de 2015, le candidat du Parti populaire (droite) avait appelé à éviter qu'El Raval devienne un « ghetto islamique $"$.

La diversification de l'offre religieuse dans ces quartiers, visible notamment par l'émergence de l'islam, ne se fait ainsi pas sans polémiques. Celles-ci rapportent en apparence de simples questions urbanistiques liées à l'usage de l'espace public. Mais ces controverses ont aussi un arrière-plan qui met en évidence les rapports entre les groupes sociaux majoritaires et minoritaires. Elles sont également l'occasion de mobiliser les ressources culturelles et institutionnelles qui permettent de classer comme illégitimes certaines formes des manifestations religieuses.

La présence de ces lieux de culte est interprétée par certains habitants comme une dégradation et la preuve que le quartier restera toujours un point d'ancrage des populations minorisées. C'est le cas notamment des nouveaux voisins attirés par la transformation urbaine et les promesses de changement faites par les administrations, qui sont aujourd'hui déçus et ressentent que l'état du quartier s'est même aggravé.

\section{MÉMOIRES, POLITIQUES ET DISCOURS SUR LA DIVERSITÉ}

Face à cette situation, les administrations de Paris et de Barcelone ont mis en place une politique qui vise différents objectifs. D'abord, en insistant sur la pertinence d'accorder aux religions minoritaires les facilités nécessaires à l'exercice de leur culte, avec diverses initiatives allant dans ce sens. À la Goutte d'Or, la mairie et la préfecture cherchent un espace pour éviter les prières de rue et réhabilitent une ancienne caserne de pompiers. À Barcelone, la mairie a mis en place un bureau des cultes qui accompagne les minorités religieuses dans les démarches nécessaires à l'ouverture d'une salle de prière ou à l'organisation de certains événements. Les festivités du Ramadan dans différents espaces d'El Raval est assurée, en partie, par l'implication directe de ce bureau. 
Parallèlement, les administrations insèrent la diversité dans les discours et les politiques de revalorisation de ces quartiers. À la Goutte d'Or, l'exemple le plus clair est l'Institut des cultures d'islam ( $\mathrm{ICl})$, conçu par la mairie. L'ICI intègre un espace dédié au culte, acheté et géré par une association islamique, aux côtés d'un autre consacré à une large offre culturelle qui vise à montrer la diversité de l'islam « au grand public ». À El Raval, la mairie organise des événements " interreligieux » avec la participation de la plupart des communautés du quartier. Des visites guidées sont également faites pour montrer par exemple « la Barcelone marocaine » aux habitants des autres quartiers et aux touristes.

Cette communication prétend aborder ces politiques et leur dimension discursive. Il s'agit de les interroger d'abord par leur relation avec les programmes de revalorisation de ces quartiers. Par exemple, la plupart des visiteurs de la partie culturelle de l'ICI proviennent de l'extérieur de la Goutte d'Or. Ceci est indissociable d'un nouveau circuit d'établissements, publics et privés, qui fleurissent dans un quartier en transformation. II s'agit également d'interroger les points critiques, car ces discours peuvent rendre invisibles les rapports sociaux établis entre les groupes du quartier. Ces politiques montrent la diversité religieuse comme quelque chose de positif sans pour autant transformer les relations d'altérité existantes. Comme le note Sylvie Tissot dans son étude d'un quartier de Boston, « l'objectif de la promotion institutionnelle de la diversité » n'est pas de questionner les rapports de pouvoir (2012).

Dans ce processus, la mémoire collective du quartier joue un rôle important et les aspects positifs sont soigneusement sélectionnés. Les discours officiels décrivent ces quartiers comme des territoires historiquement accueillants et exemplaires du vivre-ensemble, à Paris, ou de la convivencia à Barcelone. Des images sont ainsi mobilisées, qui reconstruisent l'histoire par la mise en scène d'aspects qui montrent une diversité harmonieuse. Dans cette perspective, sont oubliées les conditions de fragilité vécues par les populations migrantes (et leurs descendants) et la relégation, pendant des années et encore aujourd'hui, à l'invisibilité et à la précarité de leurs pratiques culturelles et religieuses. L'altérité structurelle à laquelle est soumise une partie importante de cette population est aussi laissée de côté. Les manifestations de cette altérité dans les quartiers, comme par exemple les expressions d'opposition à l'ouverture de mosquées ou les agressions à caractère raciste, sont en partie supprimées des récits officiels.

\section{REGARDS CROISÉS}

Cette communication porte un regard croisé sur les deux villes. II s'agit d'analyser les traits communs et divergents de ces discours et de ces politiques sur la diversité. Même à l'échelle locale, il n'est pas possible de négliger l'influence des cadres nationaux et de leurs ressources culturelles et institutionnelles. Le cadre normatif de la régulation des cultes est ainsi questionné, qui présente de fortes différences entre l'Espagne et la France. Mais aussi les modèles revendiqués par ces États à l'heure d'intégrer les minorités culturelles et religieuses qui les habitent : l'assimilationnisme assumé en France, et l'interculturalisme (sinon multi-) de plus en plus en vogue dans les administrations en Espagne.

La politique urbaine centre également ce regard croisé, car les processus de stigmatisation, puis de transformation et de valorisation des quartiers populaires (ainsi que les cadres juridiques qui les soutiennent) présentent de fortes ressemblances dans les deux pays. Pour recontextualiser les deux cas étudiés, nous tournons le regard plus particulièrement vers la place que la diversité culturelle et religieuse occupe dans les politiques urbaines contemporaines. 
Les données qui nourrissent cette communication proviennent d'une enquête de terrain menée dans le cadre d'une recherche doctorale structurée en deux phases principales dans le temps et l'espace : de mai 2016 à avril 2017 à la Goutte d'Or, et de mai à septembre 2017 à El Raval ${ }^{4}$. Sur le terrain, la méthodologie mise en place a répondu à la nécessité d'appréhender le caractère socio-historique des processus de transformation et de revalorisation urbaine. D'abord, l'analyse documentaire a permis de définir puis d'examiner les politiques publiques mises en place dans les deux quartiers. Dans cette perspective, nous avons rassemblé les plans urbanistiques et de traitement de la diversité, ainsi que divers matériaux produits par les administrations, par des collectifs et des associations. Finalement, ont été rassemblés les comptes-rendus de réunions publiques comme les conseils d'arrondissement ou les espaces dits de participation, afin d'appréhender la nature des débats, les prises de position et les discours des acteurs politiques et citoyens.

En complément de l'analyse documentaire, nous avons effectué des entretiens semistructurés auprès d'une diversité d'acteurs, constituant un corpus provisoire de 41 personnes. Une partie regroupe les responsables politiques et le personnel technique des administrations municipales (8 entretiens). Les habitants des quartiers forment l'autre partie du corpus avec 33 entretiens. Parmi eux, des militants associatifs et des commerçants, mais aussi des habitants rencontrés lors de différentes activités et par effet « boule de neige ». Par ces entretiens, il est possible d'aborder la perception des processus de transformation urbaine mais aussi de la diversité culturelle et religieuse. Les entretiens permettent également d'appréhender une mémoire collective contradictoire entre les différents groupes d'habitants en fonction de leurs appartenances et leurs trajectoires vitales et résidentielles, mais également entre ceux-ci et les discours mis en avant par les administrations.

Finalement, et dans le but de compléter les entretiens et l'analyse documentaire, plusieurs espaces ont fait l'objet d'observations spécifiques. Celles-ci ont eu lieu à l'occasion de différentes activités liées à la promotion de ladite diversité, comme des visites guidées ou la célébration de certaines festivités religieuses. Sur le terrain parisien, des observations plus régulières ont été effectuées au sein de l'Institut des cultures d'islam.

\section{RÉFÉRENCES}

Bacque M.H., 2006, « En attendant la gentrification : discours et politiques à la Goutte d'Or (1982-2000) », Sociétés contemporaines, 2006/3, $n^{\circ} 63$, p. 63-83.

Fernandez M., 2014, Matar al chino. Entre la revolución urbanística y el asedio urbano en el barrio del Raval de Barcelona, Barcelona, ed. Virus.

Gole N., 2015, Musulmans au quotidien. Une enquête européenne sur les controverses autour de l'islam, Paris, La Découverte.

Horta G., 2010, Rambla del Raval de Barcelona: de apropiaciones viandantes y procesos sociales, Barcelona, EI Viejo Topo.

Lamont M., 2000, The dignity of working men. Morality and the boundaries of Race, Class, and Immigration, New York, Russel Sage Foundation.

Milliot V., 2015, « Remettre de l'ordre dans la rue. Politiques de l'espace public à la Goutte d'Or (Paris) », Ethnologie française, 2015/3, $n^{\circ} 153$, p. 431-443.

Morovich B., 2014, « Entre stigmates et mémoires : dynamiques paradoxales de la rénovation urbaine », Articulo, 2014/5, numéro spécial [en ligne : articulo.revues.org/2529 consulté le 28 août 2017].

4 À ce jour (novembre 2017), des incursions ponctuelles continuent sur les deux terrains dans le but d'élargir le corpus d'entretiens et renforcer l'information des différentes hypothèses de la recherche. 
Tissot S., 2012, « Les métamorphoses de la domination sociale. La bourgeoisie progressiste et les quartiers populaires », Savoir/Agir, 2012/1, n 19, p. 61-68.

Toubon J.C., Messamah K., 1990, « Coexistence et confrontation dans un quartier pluri-ethnique : le cas de la Goutte d'Or », Sociétés contemporaines, n 4, p. 37-50.

\section{L'AUTEUR}

\section{Víctor Albert Blanco}

Université Paris 8 St-Denis - CRESPPA-GTM

valbert.blanco@gmail.com 